Recebido: 20.07.2012 Aprovado: 09.09.2012

\title{
Bases de la transformación de el sujeto en proceso intentada por Norbert Elias*
}

1. Professora da Universidad Nacional de Colombia. E-mail: vweiler@unal.edu. co

* Revisão técnica realizada por Anália Soria
Vera Weiler ${ }^{1}$

Resumen: En el centro de interés de este artículo se encuentra la pregunta sobre cómo enfrentó Norbert Elias la incertidumbre que sobre el conocimiento surgió desde el descubrimiento de que el conocimiento no es independiente de "el sujeto" que lo construye. Una teoría totalmente nueva, como Elias, según sus propias palabras, se había propuesto, exigía levantar de "el sujeto" productor del conocimiento y de todas las formas históricas de la vida humana, el velo del misterio inescrutable que en la tradición filosófica se preservaba. Había que investigar cómo y bajo que condiciones empíricamente constatables, adquieren los seres humanos realmente conocimiento del mundo que les sirve para vivir. Esta tarea la asumió, en gran medida, la psicología del desarrollo que se formó desde comienzos del siglo XX. De ahí que la pregunta inicialmente planteada en este artículo se traduce en la indagación por la relación entre los propósitos cognitivos y la estrategia de investigación que Elias presenta, con la revolución ontogenética producida por la psicología del desarrollo. La exposición está centrada en el periodo en que se forma la idea del desarrollo psicogenético, eje de la sociología de Elias, que es en la historia de la psicología un periodo de importantes avances sobre el problema del desarrollo humano.

Palabras clave: Norbert Elias, Sujeto, Teoria de Conocimiento, Psicología del Desarrollo, Ontogenese, Desarrollo Humano.

\section{Introducción}

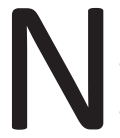

orbert Elias intentó desarrollar "un tipo de teoría...diferente de lo que tradicionalmente se considera una teoría" y, precisamente a este respecto sentía que no había logrado hacerse entender (ELIAS, 1995a, p. 87). El pensamiento que crea el tipo de teoría que él quería superar procede "como si todos los "objetos" de nuestra reflexión, incluidos los propios hombres, fuesen en principio meramente objetos, no sólo sin movimiento, sino también sin relaciones (...)" (ELIAS, 1995b, p. 135). Las conceptualizaciones en este pensamiento se construyen como si se refirieran a sustancias inmutables (ELIAS, 1996b, p. 139). Para determinado nivel de integración del universo esto puede ser adecuado. Para el ámbito socio-humano no lo es. Éste representa un nivel de integración 
que, a juicio de Elias, demanda otra manera de pensar y otro tipo de investigación (ELIAS, 1995a, p. 125-26). Pero las teorías sociológicas, Elias también las veía construidas como si los seres humanos se pudieran entender como cuerpos físicos y las relaciones entre ellos como las que se presentan entre las bolas de billar, una tendencia reforzada por la separación entre actor y acción en la estructura recurrente del lenguaje (ELIAS, 1995b, p. 134-35). De ahí que el tipo de teoría hacia el que se dirigieron sus esfuerzos involucrara la reorganización simultánea del pensamiento y del lenguaje.

La sociología procesual surgiría en la medida en que se pudiera admitir que "El hombre es un proceso" (ELIAS, 1995b, p. 142). La noción del ser humano como proceso que comienza en la más temprana infancia de cada ejemplar de la especie contradice los preceptos sobre los cuales está construida la epistemología tradicional, aquella que sostiene las teorías del tipo que Elias se propuso superar. El propósito de este escrito es poner de presente adelantos de la psicología del desarrollo que fueron importantes para la meta que Elias se trazó. Nos enfocamos especialmente en la noción procesual de "el sujeto" porque el punto crítico del hombre proceso se refiere al espíritu de este objeto. Y este es el asunto crucial del disenso entre Elias y la fundamentación epistemológica convencional de la sociología.

El único autor que ha insistido sistemáticamente en la idea de que Elias representa una auténtica ruptura epistemológica es Richard Kilminster, el editor de la Teoría del símbolo, el último de los libros de Elias publicado en vida del autor. De acuerdo con su diagnóstico, la recepción de Elias no habría asumido las consecuencias que tal ruptura implica. Esto significa que la obra de Elias habría sido asimilada a premisas que sostienen el tipo de teorías que él quiso superar, como la de una constitución psíquica idéntica siempre, que es incompatible con una sociología procesual. El hecho de que, entre investigadores que se sienten inspirados por la obra de Elias, la teoría que representa hoy los más significativos adelantos de una sociología procesual (DUX 1982; 2000; 2012) hasta ahora haya pasado prácticamente desapercibida, es coherente con el diagnóstico ya mencionado. Esta teoría, - la teoría histórico-genética de la cultura elaborada por el sociólogo alemán Günter Dux - aprovecha ampliamente la revolución ontogenética iniciada por la psicología del desarrollo anterior a la Segunda Guerra Mundial y sin ella no se puede entender. Que el eje psicogenético de la teoría de Elias se hace comprensible en la medida en que se le considera junto con la teoría de Piaget, el más famoso de los psicólogos del desarrollo, lo ha mostrado hace unos cuantos años el sociólogo alemán Georg W. Oesterdiekhoff mediante la comparación de ambas teorías (OESTERDIEKHOFF, 2000). Pero desafortunadamente este autor está convencido de que Elias tomó nota de la psicología del desarrollo y en particular de Piaget solamente en avanzada edad. Esto 
es un error. Me propongo corregirlo, cosa que exige restablecer el vínculo de la orientación procesual de la sociología de Elias con una tradición marginalizada de la psicología. Lo que a mí me parece pertinente al respecto, complementa lo expuesto recientemente por Norman Gabriel sobre el tema, ciertamente con un énfasis algo distinto al mío (GABRIEL, 2011; WEILER, 2011).

Mi tesis es que los avances que se dieron en los primeros decenios del siglo XX en la psicología del desarrollo fueron parte constitutiva de la sociología de Elias y que, para asumir las implicaciones radicales de ésta, ayuda tenerlos en cuenta. Inicialmente basta recordar que Elias en muchas ocasiones remitió a los niños a veces a los muy pequeños - y a la psicología infantil. En este terreno se había pasado a investigar cómo llegan los seres humanos efectivamente a conocer cuando Elias presentó su examen doctoral en psicología y filosofía como materias principales (ELIAS, 1995a, p. 42 y 44).

Voy a enfatizar aquellos puntos que me parecen relevantes para el concepto del desarrollo psicogenético de Elias. Algunas citas de textos de pioneros de la psicología del desarrollo pueden evidenciar importantes coincidencias. Publicadas, por fin, las reflexiones críticas de Elias sobre la idea freudiana de la sociedad como algo opuesto a las condiciones naturales de los seres humanos (JOLY, 2010), quizás se pueda contar con una renovada disposición general a considerar, para el caso que nos interesa, contribuciones adicionales a las psicoanalíticas en lo que se refiere a la psicología. No deben disuadirnos los comentarios críticos de Elias sobre la orientación dominante en esta disciplina especialmente desde el ascenso del conductismo.

Justamente en los años de formación intelectual de Elias, en la psicología del viejo continente, estaban tomando la delantera las investigaciones comparadas sobre los niños, con repercusiones sobre la orientación de la teoría psicológica en general. Todo daba a entender que los avances logrados serían la base de la reorientación de la psicología como una ciencia del desarrollo que tendría consecuencias también para el norte cognitivo de las demás disciplinas ocupadas del hombre.

2. En este apartado se integran partes de una versión revisada de Weiler 2011.

\section{Psique como objeto de formato procesual ${ }^{2}$}

Norbert Elias insistió tempranamente en que su libro sobre El proceso de la civilización no se podría entender sino bajo la condición de que los cambios ocurridos en la estructura psíquica de los hombres a lo largo del tiempo fuesen reconocidos como proceso efectivamente observable. A sus ojos, estos cambios debían asumirse como legítimo objeto de estudio. Así se lo indicó ya en 1939 a Raymond Aron, una eminencia entonces de la sociología francesa 
(WEILER, 2008, p. 794), y esta indicación seguramente aplica a toda su obra. Pero el objeto de formato procesual al que se refería Elias como tal aún hoy no está generalmente admitido, para gran parte de los científicos sociales sencillamente no existe.

A este respecto las cosas todavía lucen como si poco hubiera cambiado desde finales del siglo XIX cuando, en razón de una convicción similar a la manifestada por Elias a Aron, y basado en sus investigaciones de primera mano sobre más de mil años de historia germánica, el historiador Karl Lamprecht (1856-1915) planteó la necesidad de asumir una estrategia psicogenética para hacer inteligible la historia. La propuesta suscitó una tormenta al cabo de la cual terminó echada en saco roto por la mayoría no solo en Alemania y no solo entre los historiadores. Si la negativa frente a la estrategia psicogenética esbozada por Lamprecht tuvo por epicentro al gremio de los historiadores alemanes, otro capítulo con tema de fondo y efectos similares se abrió un poco más tarde en Francia. Allí, comenzó en los años veinte, por iniciativa de antropólogos, filósofos y sociólogos galos, una ofensiva contra la obra de Lévy-Bruhl (1857-1939) que pronto derivaría en un veto abierto y duradero. Era una reacción a los libros en que Lévy-Bruhl había procedido a sistematizar el acumulado etnográfico disponible con el propósito de establecer las características propias de la mentalidad primitiva. Su hipótesis estipulaba que ésta presenta diferencias significativas con la mentalidad general de las sociedades industriales. Pero en las ciencias sociales gobernaba un espíritu inclinado hacia el supuesto, heredado de la filosofía tradicional, de una psique humana siempre igual. La psique, pensada así, es el equivalente a un atributo constante de la especie. Las reacciones a Lamprecht y Lévy-Bruhl nunca revisadas de fondo dan una idea de cuán difícil resulta llegar a concebir al hombre como proceso.

Elias debió conocer la propuesta psicogenética de Lamprecht. Saltan a la vista las coincidencias acerca del proceso psicogenético y su centralidad para la comprensión de la historia (y del presente). "La distancia psíquica" se llama un capítulo de la Introducción al pensamiento histórico publicada en 1912 por Lamprecht que bajo este título tematizaba la distancia psíquica entre épocas de la historia. Coinciden Lamprecht y Elias ampliamente sobre las tendencias del cambio en las estructuras psíquicas en el largo plazo al considerar:

- que estas estructuras como un todo se manifiestan en un hábito mental general característico de los respectivos niveles de desarrollo históricosocial,

- que sus transformaciones presentan un orden secuencial con una dirección observable y

- que la tendencia de largo plazo presenta cambios hacia afectos más matizados y equilibrados, asociados a cambios en la manera de pensar (WEILER, 2010, p. 248-251). 
Es cierto, a Elias no se le conoce comentario directo a Lamprecht. Pero resulta difícil creer que lo haya ignorado no solo por las coincidencias que se registran sino también por las reacciones estruendosas que suscitaron las propuestas del historiador.

En lo referente a Lévy-Bruhl hay pruebas incontrovertibles de que Elias no solo conocía la obra sino también de que no compartía la opinión dominante sobre la misma. En su intercambio epistolar de 1939 con Raymond Aron, Elias se refirió a Lévy-Bruhl en términos que indican que ya en este tiempo se hallaba en completo desacuerdo con la posición asumida por los colegas parisinos en relación con los estudios sobre la mentalidad primitiva (WEILER, 2008). En concepto de Elias los trabajos de Lévy-Bruhl contribuían significativamente a establecer como auténtico objeto de investigación los cambios psíquicos observables a lo largo del tiempo.

Los manuscritos de mediados de los años sesenta relacionados con Lévy-Bruhl (WEILER, 2008) dejan claro que Elias mantenía su apreciación inicial de este autor $y$, de paso, sobre los problemas que presentan unas ciencias sociales que no han vencido en su desarrollo el umbral hacia el reconocimiento de unos cambios en la psique humana a lo largo del tiempo como proceso realmente observable. La hipótesis de trabajo de Lévy-Bruhl, así como el hecho de que éste hubiera sabido mantenerla intacta a lo largo de su investigación, le parecía a Elias, en clara oposición a la opinión dominante, de suma importancia. Porque, como ya hemos registrado, en el campo en discusión sucedía con regularidad que la hipótesis central se derivaba directamente de un supuesto comúnmente aceptado - el sujeto en esencia es siempre igual -, de manera que la interpretación de los resultados empíricos solía quedar circunscrita de entrada a dicho supuesto. A ojos de Elias tal mecanismo gobernaba las críticas en que se basaba el veto contra Lévy-Bruhl en tanto que éste supo evadir la circularidad característica del mismo (WEILER, 2008, p. 811-812).

Los comienzos de la revolución ontogenética

\section{Antecedentes}

Lo que quiero mostrar es el soporte que recibió Elias de la psicología del desarrollo que tomó forma entre el segundo y cuarto decenio del siglo XX. Comienzo con unas notas introductorias al campo. La psicología se originó en la filosofía; como actividad con pretensiones explícitamente científicas, inició su camino en el siglo XIX, bajo el impacto del creciente prestigio social de las ciencias 
naturales y, desde luego, de la teoría biológica de la evolución de las especies. Por unas cuantas décadas no había psicólogos en el sentido que la profesión alcanzaría posteriormente. Eran filósofos dedicados a cierto tipo de problemas. En las universidades europeas de comienzos del siglo XX, por lo general, formaban parte de las facultades de filosofía y solían ocupar cátedras de filosofía en combinación con algún otro campo. Al tiempo con la continuidad de la tradición filosófica se hacía sentir un fuerte impulso empírico, experimental. Su manifestación más visible fue la creación de una serie de laboratorios en distintos países, comenzando por el de Wilhelm Wundt (1832-1920) en la Universidad de Leipzig (Alemania), en 1879. Así surgió una suerte de filósofo experimental. Desde hace tiempo esto luce difícil de asumir. Aparece como una contradicción en los términos. Sin embargo, para los psicólogos de comienzos del siglo XX no lo fue. Ciertamente, la psicología desplegó la nueva vocación inductiva experimental y comenzó a prosperar precisamente en donde lo había hecho la filosofía, tanto como si el suelo para ello lo hubiera preparado la filosofía misma. Sea esto como quiera, para hacerse una idea de cómo nació la psicología del desarrollo en particular y de los resultados que produjo con anterioridad a la Segunda Guerra Mundial ha de tenerse en cuenta que se trata de un desarrollo en el cual confluyeron la erudición filosófica y el impulso empírico experimental. Este impulso se suele suponer como factor decisivo en el proceso de gestación de la psicología moderna. Y hasta cierto punto esto es acertado. Pero no se debe subestimar el peso de la formación filosófica de los psicólogos de la época, por cuanto ella implicó una familiaridad con ideas ensayadas a lo largo de la historia sobre la psique y un conocimiento de los debates habidos entorno a ellas, que no se puede tomar por cosa habitual en los laboratorios de la psicología de hoy.

La psicología sobre la cual se centra la atención de este artículo, es aquella que se plantea el problema del desarrollo en términos de un proceso genético, en que sucesivos estadios con una característica organización psíquica general surgen, uno de otro, y donde la investigación trata de resolver cómo y por qué lo hacen.

No hay duda de que la teoría biológica de la evolución ayudó a preparar el suelo del que surgió este concepto de desarrollo en psicología. Muchas referencias explícitas lo dejan en claro. Pero las pruebas también enseñan que por sí solo el enfoque biológico evolutivo no obliga a concebir los fenómenos psíquicos en su conjunto como un proceso de desarrollo en los términos aludidos. Es cierto, tampoco hay filosofía que lo haga. Pero no se puede pasar por alto una relación temática específica entre la filosofía y la psicología. A fin de cuentas ésta se ocupa de asuntos que por más de dos milenios han sido resorte y corazón de la filosofía. En primer lugar la epistemología. Y en la medida en que hace bien su trabajo se va convirtiendo en su rival. Para esto cuenta con un legado de 
ideas filosóficas precisamente en la materia que hereda, para transformarla eventualmente. Puede servir de ejemplo de ilustración el espíritu mundi postulado por Hegel. Envuelto en la especulación metafísica idealista, contiene la osadía de plantear un desarrollo espiritual intramundano, asociado a períodos de la historia de la humanidad que representan distintas formas de organización social. La lógica dialéctica hegeliana también ha facilitado la superación de las restricciones que a la posibilidad de pensar el desarrollo opone, en la lógica clásica aristotélica, la condición de identidad de las cosas con ellas mismas. En ocasiones los psicólogos genéticos mismos remiten directamente a las ideas sobre la dinámica del espíritu, de Hegel (WERNER, 1953, p. 28). El psicólogo Jaan Valsiner, en su estudio sobre la historia de la psicología evolutiva soviética, afirma que ésta habría despegado en los años veinte bajo la influencia notoria de la dialéctica de Hegel, Engels y Marx (VALSINER, 1988). Norbert Elias, por su parte, subrayó el mérito de la no-identidad en sus reflexiones acerca de la hipótesis que guió los estudios de Lévy-Bruhl sobre la mentalidad primitiva (WEILER, 2008, p. 811).

Para entrar en materia conviene aclarar algunos términos. La voz psicología del desarrollo se emplea con distintos matices: unas veces como sinónimo de psicología de los niños (infantil) y otras para hablar de una manera de concebir el desarrollo psíquico en general. Esta es la que a veces también se llama genética y que implica la idea de cambiantes cualidades o niveles de organización que surgen unos de otros. La moderna psicología de los niños nació en los años ochenta del siglo XIX. Una figura importante en su creación fue William Preyer (1841-1897), a la sazón catedrático en Jena (Alemania). A comienzos de 1880, Preyer presentó, en la Sociedad Científica en Berlín, una conferencia con el título Psychogenese (Psicogénesis) y en su libro Die Seele des Kindes (El alma del niño), cuya primera edición data de 1882, se refiere a las observaciones diarias del desarrollo de sus hijos durante los primeros dos años de vida en términos de hechos psicogéneticos. El orden cronológico en que van apareciendo novedades en el desarrollo de los niños es vital para la lectura psicogenética de lo observado. Esto da cuenta de la orientación psicogenética con que nació la psicología de los niños. Pero es preciso notar que en los estudios posteriores de los niños este énfasis no siempre se presenta, así como el concepto de desarrollo que se advierte en ellos puede variar considerablemente del genético.

Los significados de los distintos términos en uso, por lo ya señalado, se traslapan; pero, en la medida en que todavía no hay otros, el contexto en que se emplean debe ayudar a establecer el matiz en cada caso. Esto aplica también para la expresión de psicología evolutiva que heredamos de traducciones que prefieren evolución a desarrollo, probablemente por la comodidad en la formación del correspondiente adjetivo. 
Paralelos notorios entre historia de la humanidad y desarrollo individual. Un punto de partida

Que la historia de la humanidad y el proceso de los niños presentan unos paralelos es una idea muy vieja. Pero sólo es un punto de partida. A comienzos del siglo XX nadie se hacía la ilusión de que ya se supiera cómo explicar tales paralelos. Se tenía conciencia más bien de que no se sabía en qué exactamente consisten. En realidad, los conocimientos empíricos eran insuficientes para aventurar apuestas teóricas de gran alcance sobre la relación entre historia individual y proceso histórico colectivo. Pero la psicología se planteaba el tema.

He aquí una cita para ilustrar en qué forma lo hicieron los psicólogos interesados en el problema del desarrollo. Los párrafos que presento son de William Stern (1871-1938), los extraigo de una obra de fundamentación filosófica de la psicología que fue publicada en 1906. Acerca de los paralelos a los cuales hemos aludido se lee lo siguiente:

En los primeros meses de su vida el individuo humano se caracteriza como lactante por la preponderancia de los sentidos elementales, de la vida de los reflejos e instintos primarios. Se encuentra en el estadio de los mamíferos. En la segunda mitad del primer año alcanza la actividad de la prensión y de muy diversas imitaciones, y con ello el estadio de los simios, los mamíferos superiores. En el segundo año de su vida el niño llega a caminar erguido y adquiere el lenguaje, así experimenta su verdadera humanización. Durante los siguientes cinco años de los juegos y de los cuentos de hadas el niño se encuentra en el nivel de los pueblos naturales. Luego se produce su ingreso a la escuela, la incorporación más rigurosa en un todo social con deberes precisos, la clara separación entre trabajo y ocio: se trata del paralelo ontogenético con los inicios de la cultura humana, con sus organizaciones estatales y económicas. En los primeros años de la edad escolar lo más adecuado al espíritu infantil son las condiciones simples de la Antigüedad y del Antiguo Testamento, los años medios registran los rasgos románticos de la cultura cristiana, y solo el tiempo de la adolescencia presenta aquella diferenciación que se corresponde con el nivel cultural de los tiempos recientes (contemporáneos). La edad de la pubertad con frecuencia ha sido denominada la época de la llustración del individuo (STERN, 1906, p. 299-300 - traducción mía). 
No hace falta estar de acuerdo en detalle con la caracterización de los periodos presentada. Lo que es preciso notar es que a comienzos del siglo XX, el tema base de la comparación presentada no es para psicólogos motivo de protesta. Entre ellos, unas analogías entre proceso filogenético y ontogenético se dan por sentado y se tiende a interpretarlas como indicio de que ambos planos están relacionados. La pregunta es cómo. Para comienzos de los años veinte, que son años muy productivos para la psicología del desarrollo en Europa continental y en la Unión Soviética, ya se están debatiendo varias teorías que tratan de responder esta pregunta. $Y$ es esta discusión precisamente un factor que estimula y orienta en no poca medida las investigaciones empíricas sobre el comportamiento en los animales, los niños, los llamados pueblos naturales y los enfermos mentales, paso a paso (KOFFKA, 1921, p. 32-35; WERNER, 1926). Son los años en que se forma la orientación científica de Norbert Elias y que él culmina, como ya se ha mencionado, como doctor en filosofía y psicología.

Proceso y la organización de la comparación regida por el moderno concepto de tiempo

De la misma manera que se advierte en el esquema presentado por Stern en 1906, los psicólogos del desarrollo en los años veinte relacionan con el proceso individual a toda la historia de la especie humana, no solo uno o varios de sus fragmentos. Dos aspectos de lo que esto significa en cuanto a la historia son dignos de ser resaltados. Primero, el horizonte de la comparación aquí es el desarrollo psíquico a lo largo de la historia de la humanidad en su totalidad. Se puede mirar a esta totalidad como un conjunto atemporal, como un conjunto de piezas dadas en el momento de nuestra observación. Pero, si de la consideración de las piezas no se elimina la dimensión temporal, si, por el contrario, esta dimensión se considera constitutiva del conjunto, este conjunto se presenta distinto. La psicología del desarrollo, que tomó cuerpo en los primeros decenios del siglo XX, asume la segunda perspectiva, la que incluye la dimensión temporal. Tal perspectiva se basa en la irreversibilidad del tiempo, y esta es una condición para que constituciones cualitativamente distintas de la psique se puedan conceptualizar como un proceso de desarrollo (VALSINER, 1993, p. 13-15). La comparación sugerida por el esquema de Stern, esto es lo que quería resaltar, es una comparación basada en el orden de sucesión en cada caso de fases cualitativamente distintas. Como se advierte en el esquema de Stern, tomar en la mira toda la historia también remite a los inicios, por lo cual los psicolólogos del desarrollo basados en la teoría de la evolución de las especies también se interesaban por los animales e iniciaron la investigación científica especialmente de la cognición de los primates. De ahí que los experimentos realizados por 
Wolfgang Köhler (1887-1967) para establecer empíricamente las habilidades cognitivas y el tipo de aprendizaje presente en los chimpancés (KÖHLER, 1917), les resultó de la mayor importancia. Elias también los conocía, en sus cursos de extensión en Londres se remitía con regularidad a ellos.

\section{Etnografía comparada y psicología infantil}

Etnógrafos y psicólogos infantiles ejercen profesiones que no se suelen confundir, sus investigaciones están dedicadas a campos que no solo son distintos sino que hoy se encuentran claramente separados. Para la psicología genética de los primeros decenios del siglo XX, existía la distinción entre las tareas de investigación especializada de cada una de estas disciplinas, así como de las especializaciones al interior de cada una de ellas. Pero, en la medida en que el método comparado era considerado como la principal herramienta de una psicología general del desarrollo, se experimentaba la necesidad de integrar el material producido por todas esas investigaciones.

La etnográfica real de Elias entendía su trabajo como una labor de la cual se esperaba alguna ilustración sobre cómo podía haber sido la vida humana en un pasado remoto, anterior al surgimiento de la religión, de la agricultura, de la escritura y del Estado por ejemplo. En esta idea se había inspirado la antropología clásica del siglo XIX; ella tuvo gran influencia en la vecina sociología y era muy común a comienzos del siglo XX concebir los datos etnográficos en este sentido.

No obstante esto, a los etnógrafos se les hacía muy difícil ver en sus informes de campo indicios de proceso de estructuras psíquicas cambiantes (VOLKELT, 1914, p. 6). La mayoría de ellos tendía a representarse la mentalidad de los grupos que estudiaba con base en la identidad duradera de la psique humana. Para una psicología genética, por otra parte, la etnografía resultaba vital, porque en caso de que existieran estructuras cognitivas inmutables a lo largo de la historia de la humanidad, esto debiera confirmarse, en primer lugar, en la comparación entre las estructuras contemporáneas y los más lejanos ancestros. Fue exactamente esta reflexión lo que motivó a Lévy-Bruhl a dedicarse al estudio de la mentalidad primitiva. Y por esta razón sus resultados eran relevantes para los psicólogos interesados en el desarrollo. Pero el interés de esos psicólogos por la etnografía no se limitaba a Lévy-Bruhl. La comparación de los datos provenientes de sus propias investigaciones con los que proporcionaba la etnografía entre ellos era usual. La comparación entre el conocimiento cada vez más preciso sobre las distintas fases del desarrollo infantil con los datos sobre las sociedades primitivas atraviesa también los estudios especializados en la percepción, el lenguaje y las formas de razonar. 
Es preciso tener en cuenta que, en la segunda mitad del siglo XIX, la etnografía se expandió rápidamente y fue bastante popular. Por largo tiempo funcionarios coloniales, misioneros y uno que otro viajero aficionado, habían sido la fuente del conocimiento disponible sobre aquellos pueblos y aquellas regiones del mundo que no eran accesibles a la experiencia directa para las personas comunes, pero de cuya existencia ya se había tomado nota. En la segunda mitad del siglo XIX ellos fueron sustituidos en la medida en que avanzaban las expediciones más especializadas en la recolección de la información etnográfica, los estudios de campo se volvieron más sistemáticos y profesionales. Surgieron numerosas revistas científicas, museos etnográficos, e Institutos de investigación. La psicología de los pueblos (Völkerpsychologie) como parte de la psicología moderna, se formó entrelazada con la etnografía desde sus inicios. Tan tempranamente como que en 1862, Wundt arguyó que la psicología general, para volverse una ciencia con sólido fundamento, requería del soporte de la psicología comparada (ésta incluye la psicología evolutiva y la Völkerpsychologie) (MÜLLER, 2005, p. 37). Y, en 1912, con el foco de atención más centrado en el desarrollo, esta psicología de los pueblos del mismo Wundt, ya era concebida en términos de una "Historia psicológica del desarrollo de la Humanidad" (WUNDT, 1912). Lo que se advierte de manera ejemplar en la evolución de la psicología en la Universidad de Leipzig de Wundt, Krueger y Volkelt, es la fuerza con que la etnografía empujaba la psicología hacia el problema del desarrollo socio-cultural. Y es precisamente en este contexto que se plantea, como problema fundamental de la psicología, la relación entre proceso individual y colectivo a lo largo de la historia de la humanidad (WUNDT, 1912; KRUEGER, 1915; NIESTROJ, 1989). En concepto de Krueger, el plano individual como el social era asunto de una psicología indivisible con un mismo concepto científico de desarrollo. Este concepto integraba tres rasgos:

1. Los cambios en forma de desarrollo son continuos.

2. Los cambios en forma de desarrollo suceden en un todo de partes cualitativamente distintas que interactúan unas con otras. La totalidad se preserva a través de todos los cambios como un sistema. La totalidad es más que la suma de sus partes porque constituye una estructura viva de síntesis. Y por ello la totalidad no puede ser comprendida sin que se considere la interacción pasada y actual de sus partes. El enfoque holístico aplica no solo al organismo individual sino también a una unidad cultural mayor que representa una realidad propia constituida por diferentes esferas culturales relacionadas (como economía, costumbres, leyes, arte, religión).

3. Los cambios en el todo presentan una dirección, y esta dirección es conceptualmente determinada por leyes estructurales cuyo 
descubrimiento es la tarea de la teoría del desarrollo. (MÜLLER, 2005, p. 38 con base en KRUEGER, 1915, p. 167-168, 202 - traducción mía)

Para avanzar con esta tarea la investigación debía hacer uso de todos los métodos disponibles. Para el estudio de los fenómenos culturales, sin embargo, en concepto de Krueger, es absolutamente indispensable el método genéticocomparativo.

Para ilustrar cómo continuó el desarrollo de estas ideas durante los años veinte con base en un ejemplo representativo, me quiero referir a Heinz Werner (18901964). Werner, después de su formación en Viena, prácticas experimentales ahí mismo y en Munich, junto a Karl Bühler trabajó, desde 1917 hasta 1933, junto con William Stern, en el laboratorio del afamado Instituto de Investigaciones Psicológicas en Hamburgo. En 1926 publicó un importante libro, conocido por Elias, Psicología comparada del desarrollo mental. Se trata del libro publicado por Werner, conocido en español como Compendio de psicología evolutiva (WERNER, 1936) o Psicología comparada del desarrollo mental (WERNER, 1965). A este libro se refiere Georg W. Oesterdiekhoff al afirmar por una parte que "...la obra de Heinz Werner (1926) se puede entender como confirmación del concepto de psicogénesis de Norbert Elias", y por otra que "Si Elias hubiera conocido a Heinz Werner, habría podido apoyarse en su obra y así no hubiera tenido necesidad de confeccionar su propio concepto de psicogénesis" (OESTERDIEKHOFF, 2011, p. 86; en la misma tónica todo OESTERDIKHOFF, 2000). En efecto, las coincidencias entre Elias y Werner merecen la mayor atención. Es por esta razón que me voy a detener en el libro mencionado. Pero hay que insistir en que es absolutamente insostenible la idea de Oesterdiekhoff de que Elias habría ignorado justamente esta obra. El libro de Werner es para los interesados en una perspectiva psicogenética una referencia obligatoria en el tiempo en que Elias elabora La sociedad cortesana y El proceso de la civilización.

Veamos de qué se trata: "Además de una introducción en la esencia normativa de la psicología genética, se describen y diferencian sus concepciones teóricas, tomando por base problemas fundamentales y concretos de la psicología comparada", decía el escueto prólogo a la primera edición (WERNER, 1936, p. VI). La primera parte del libro está dedicada a la exposición del método y objeto de una psicología general del desarrollo. Al igual que Felix Krueger (1915), Werner ve en la formulación de leyes generales de la evolución psíquica el objetivo de la psicología. Tal objetivo le parece alcanzable, en la medida en que el trabajo en todas las ramas de la psicología adopte una orientación genética. Así, la diversidad de los objetos de estudio de las distintas psicologías especiales (individual, colectiva, animal, infantil, etc.) no daría lugar a resultados incompatibles sino que, por el contrario, todos los resultados se habrían de integrar en una psicología general, que se entiende como ciencia genética comparada. 
Esto significa que la idea que Werner tiene de la psicología genética (evolutiva, del desarrollo) no es la de una nueva rama especializada de la psicología sino más bien la de una reorientación general en la manera de pensar lo psíquico. La reorientación genética, como la plantea Werner, concierne a la transformación de objetos estáticos en objetos procesuales (es decir, objetos que tienen forma de procesos); así, la transformación de objetos estáticos en objetos procesuales, a la vez que la idea del cambio, aparecen como aspectos de una misma transformación en el pensamiento de los sujetos que buscan comprender la psique. Son aspectos que se condicionan mutuamente, de suerte que no se pueden conseguir objetos genéticos y conservar a la vez una concepción del cambio que obedezca a la lógica de la identidad. No hay objetos genéticos sin proceso genético.

En esta idea de lo que exige el desarrollo de la nueva psicología genética de parte de los sujetos, se encuentra la cercanía fundamental entre la visión genética de las cosas de Werner y la idea de una sociología procesual de Elias. De la misma manera en que aquel plantea una reorientación general de la manera de pensar el ámbito psíquico, Elias concibe la sociología como una manera de concebir todo el ámbito socio-humano en términos de una lógica procesual. Y, por esta misma razón no quería ser entendido como inventor de una nueva rama especial de la sociología, cual creador de un nuevo objeto en las estructuras en que se venían creando objetos.

Vuelvo sobre Werner. De acuerdo con él, la perspectiva genética permitiría superar la concepción cuantitativa del desarrollo que va de la mano de la idea de la psique como compuesto de manera atomística y/o mecanicista de unos elementos divisibles pero en esencia inmutables. En tal concepción Werner ve la base, por ejemplo, de todas aquellas representaciones en las cuales el proceso del niño aparece como una serie de adiciones de elementos que se encuentran en el adulto y el espíritu de los pueblos como una suma de unos elementos individuales.

En perspectiva genética, el desarrollo, a juicio de Werner, no se presenta como problema de crecimiento cuantitativo $y$, en consecuencia, no se puede comprender con base en técnicas cuantitativas, sino que se plantea en términos de niveles de organización interna de unidades vivas reales. Su estudio entonces plantea dos problemas fundamentales: el de la estructura peculiar de las distintas fases (cortes transversales), y la determinación de la dirección evolutiva ("el sentido y la tendencia responsable de la orientación de tales fases en la constitución de una relación genética supraordenada").

Así pues, la determinación de la estructura de los períodos mentales aislados, ya se trate del desarrollo individual, 
del infante o del adulto, o de la humanidad en general, es un problema genético, y el otro es la determinación de la forma evolutiva y de la dirección que sirve de eje de referencia y orientación de tales fases (WERNER, 1936, p. 5).

La dirección general del desarrollo, para Werner, va de menor a mayor diferenciación entre las partes que integran un todo jerárquico de suerte que, al tiempo que va aumentando el grado de especialización de las partes, va aumentando el grado de su dependencia del nivel de integración del conjunto. Así que Werner advierte que en el proceso del desarrollo psíquico se conserva la dirección general que registra la evolución de los organismos. Su continuación en la historia de la cultura asoma en la segunda parte del libro, cuyo tema central es la estructura psíquica primordial o primitiva. Antes de pasar a este tema más concreto quiero mencionar dos razones por las cuales Werner considera necesario adoptar una perspectiva genética en psicología. Una apunta a la necesidad de hacer inteligible el hecho de la diversidad de formas o tipos. Para ilustrar de qué tipo es el reto que se plantea Werner remite a la experiencia de la biología. En este campo el concepto de la evolución no habría surgido como el producto de la especulación sino

(...) derivado de la necesidad de comprender la multiformidad graduable de las formas biológicas, disponiéndolas de acuerdo con las fases genéticas a que corresponden. El principio de la evolución es, por consiguiente - dice - imprescindible para todo conocimiento biológico inteligente.

Algo análogo sucede en la psicología evolutiva: existen aquí tipos psíquicos, considerados empíricamente, que aparecen graduados y susceptibles de seriación formal, en cuanto a su naturaleza esencial y a sus propiedades funcionales. Si, pues, no queremos tampoco contentarnos aquí con la mera constatación de esta multiformidad, es necesario que orientemos las diversas formas psíquicas agrupándolas en un conjunto significativo. Una tal tarea de integración sólo puede realizarse acudiendo al principio evolutivo (WERNER, 1936, p. 4-5).

El argumento central es el mismo que el que años atrás esgrimió Lamprecht al indicar en qué dirección debía orientarse el pensamiento histórico para poder enfrentar el tema de la diversidad de las formas histórico culturales de la vida humana. De manera similar a la de Werner, este historiador había echado mano de la experiencia que ofrecía la adopción de un punto de vista evolutivo en la biología (WEILER, 2010).

Para Werner, además, el problema de la diversidad está ligado a la comprensión 
del hombre actual. Este es el segundo punto de su sustentación de la necesidad del punto de vista genético que quería comentar. Werner enfatiza este punto donde trata de ilustrar en qué medida la perspectiva genética modifica no solo las posibilidades de explicar la diversidad de formas en el tiempo sino también afecta la visión que tenemos de nosotros mismos. Recurro a una cita extensa que permite ver las relaciones en que Werner ve el asunto. Acerca de los adultos modernos se registra lo siguiente:

Su estructura psíquica se caracteriza, no por la existencia de un nivel uniforme, sino por la imbricación de varios estratos genéticos, y por ello incluso el individuo aislado nos muestra, cuando se le considera genéticamente, diversos valores en sus manifestaciones expresivas, que corresponden a fases determinadas de la evolución ocurrida.

La creencia de que la vida psíquica normal del hombre civilizado muestra una sola esfera mental se halla relacionada íntimamente con un especial modo psicológico de considerar la estructura psíquica, a saber: con la denominada psicología asociacionista o elemental, que intenta reducir todos los sucesos psíquicos a elementos rígidos de los cuales derivarían la percepción y la acción, el sentimiento y el pensamiento. El modelo atómico sirve en este tipo de psicología de norma para la concepción de la estructura psíquica, y así se desarrolla una dirección mecanicista, a la cual se opone el criterio deducido de la consideración psicogenética. Según ésta, el europeo es multifacético, no posee una sola actitud mental ni un solo modo de vivenciar y reaccionar, sino que un mismo y único hombre observado en diversos momentos nos puede aparecer como representante de diversos periodos evolutivos. (WERNER, 1936, p. 31-33)

La segunda parte del libro de 1926 está dedicada al estudio de las actividades mentales primitivas. El objetivo es la caracterización de las estructuras psíquicas primeras que se encuentran a nivel individual y a nivel colectivo, como un todo orgánico en el sentido arriba comentado. Para mediados de los años veinte ya se cuenta con un gran volumen de investigaciones especializadas sobre los niños y sobre grupos humanos primitivos, sobre los animales y enfermedades mentales, que se ofrecen para la sistematización comparativa. El interés en la caracterización de un estado del desarrollo psíquico como un todo, lleva a Werner a integrar registros que suelen tratarse por separado: comienza por la difusa organización sensorio-motriz, perceptual y afectiva; estudia las representaciones primitivas igualmente difusas y típicamente sincréticas, las nociones 
primitivas de tiempo y espacio, el mundo de las acciones que se presentan siempre ligadas a situaciones concretas, los procesos primitivos del pensamiento $y$, finalmente, la estructura de la personalidad primitiva. En los casos bajo inspección comparativa, Werner encuentra una sola estructura global que se caracteriza por un mismo grado de indiferenciación entre las actividades de percibir, sentir, actuar y pensar. Ninguna de estas funciones, que tradicionalmente se tratan como claramente distinguibles, en la mentalidad primitiva (de niños y adultos primitivos) se podría entender por separado. En el nivel en que está interesado Werner, ellas acaso se muestran como aspectos interdependientes en un mismo nivel de organización. El rasgo estructural que más claramente caracteriza este nivel es la cualidad global de las experiencias para los sujetos. El mundo que éste experimenta y en el que actúa sin distinguir es un mundo de sensaciones globales. No hay una clara dualidad entre sujeto y objeto, de hecho no existen objetos independientes en relaciones espacio-temporales sino situaciones que los sujetos experimentan en tanto experimentan su propio estado general. Este estado general es para la mentalidad primitiva la medida de todo e interviene activamente en la constitución del mundo como es para esta mentalidad. Este estado general, por ejemplo en la forma que nosotros llamamos afectos en los procesos de percepción, moldea activamente lo que se percibe. De manera que lo que se percibe es una suerte de situación global referida al ser que la experimenta sin conocerse a sí mismo como sujeto y por tanto sin conocimiento de la parte que juega él mismo en la constitución de la situación global que experimenta. El mundo de la mentalidad primitiva es, pues, un mundo de acción y eficacia bajo las condiciones en que no hay distinción estable entre sujeto y objeto. Las prácticas de niños y grupos humanos que presentan tal estructura psíquica y de personalidad se rigen precisamente por esta estructura y pueden ser explicadas en gracia a ella. El ejemplo más conocido es la magia.

Es evidente que el análisis de Werner confirma los principales resultados de los estudios de Lévy-Bruhl en cuanto a las sociedades más simples. Werner cree inapropiado referirse a la mentalidad primitiva como pre-lógica, le parece que la estructura que ella presenta constituye más bien una lógica propia. Pero en la medida en que a Lévy-Bruhl mismo el término en discusión finalmente no le parecía adecuado y, ante todo en gracia a las amplias coincidencias de contenido entre su trabajo y el de Werner, no restan diferencias de fondo entre ambos autores a propósito de los primitivos.

La novedad del trabajo de Werner consiste en una significativa ampliación del horizonte comparativo que se beneficia de su familiaridad con los avances en los distintos campos de la psicología experimental, incluida la infantil. Muchas veces remite a las investigaciones adelantadas en su propio instituto en Hamburgo. El resultado es la constatación de la similitud formal (para usar la terminología 
del autor), de las estructuras psíquicas primitivas a nivel colectivo (histórico) y a nivel ontogenético, registrado en los niños. En este sentido se continúa hablando de la estructura primitiva en singular. Werner habla de similitudes formales para dejar claro que las similitudes encontradas no ponen en tela de juicio que el adulto primitivo sea efectivamente adulto y que esto lo distingue claramente de cualquier niño del pasado y del presente. Para explicar el asunto, Werner remite a la plasticidad de los niños, al hecho de que los niños siempre crecen en un mundo de adultos, mientras los adultos en toda parte viven en su propio mundo que es, pues, de adultos. No obstante estas precauciones, Werner no escapa de la crítica de quienes creen que las similitudes encontradas se pueden despachar acusando de prejuicios ideológicos (infantilización de los salvajes) a quien las encuentra (JAHODA, 1999, p. 185-192).

Este tipo de crítica representa un gran problema no solo para la integración de Werner sino de los hallazgos de importantes sucesores de él a los conocimientos básicos de las ciencias sociales. El mismo estigma ideológico o moral compromete nuestra comprensión de la sociología de Elias, muy en particular la del eje psicogenético de la teoría que esbozó. Desde la gran ofensiva crítica liderada por el antropólogo Anton Blok contra la idea de los primitivos incorporada en el esbozo teórico de Elias (WILTERDINK, 1984; MENNELL, 1992; WEILER, 2011, p. 120-23) el tema por lo común se evita, excepcionales intentos de réplica se enfocan sobre asuntos de segundo plano (LISTON \& MENNELL, 2009). La práctica evasiva forma parte de la recepción a medias de Elias, sobre cuyos efectos desde una perspectiva un poco distinta a la que aquí nos ocupa, Richard Kilminster recientemente ha vuelto a llamar la atención (KILMINSTER, 2011).

Las similitudes estructurales encontradas por Werner en lo fundamental se han confirmado. De modo que para el caso en discusión se plantea la necesidad de esclarecer la aparente impermeabilidad de la teoría frente a las evidencias empíricas que parecieran, por otro lado, exigir su revisión.

Los contemporáneos de Werner, que compartían esfuerzos en la construcción de una psicología genética, entendían lo hecho por éste como confirmación de la idea que varios de ellos habían formulado a propósito de que los avances en el estudio de los niños contribuirían a esclarecer problemas de la historia de la humanidad, en especial los relacionados con las fases tempranas de la misma (STERN, 1907; BÜHLER, 1918; KOFFKA, 1921).

Cuando el psicólogo Gordon Allport (1897 - 1967) presentó, en 1940, el libro de Werner a los psicólogos norteamericanos, les advertía que la obra suponía superar su predilección cultural por el enfoque mecanicista y aprovechar que gracias a los desastres políticos habidos en Alemania les había llegado la avanzada europea de la nueva psicología orgánica. Recomendaba la lectura del 
libro de Werner a los profesores y estudiantes de psicología social, psicología infantil y psicopatología y estaba seguro de que también los antropólogos culturales lo iban a acoger con gran interés y beneficio (WERNER, 1940, p. XI; 1965, p. 12). Pero el reconocimiento que Werner recibió en EEUU, país en que se había exiliado, se debe mucho más a su labor como psicólogo experimental y prácticamente no guarda relación con los avances ofrecidos por él para una teoría del desarrollo humano (VALSINER, 2005).

Elias apreciaba mucho a William Stern (GLEICHMANN, 1987, p. 410). Para Stern, fueron los trabajos que se estaban realizando en la perspectiva de una psicología del desarrollo , lo que más daba la impresión de que la psicología estaba cambiando de rumbo. Los veía en gran medida afines a su propio enfoque personalista. El mérito estratégico de la psicología del desarrollo en su opinión, era la conceptualización "del curso del desarrollo psíquico como un solo proceso estructurado".

Entre los avances más recientes, Stern resaltaba enfáticamente la Introducción de Werner (1926), como primer resumen sistemático - desde los inicios de Felix Krueger, años atrás - de una psicología del desarrollo del tipo que enfrenta los problemas arriba señalados, el de las formas psíquicas primitivas en primerísimo lugar. En el paso siguiente se planteaba, en concepto de Stern, la tarea de establecer con mayor precisión la estructura característica de los diferentes estadios del desarrollo. Entre los trabajos que apuntaban en esta nueva dirección mencionaba los estudios de Jean Piaget sobre el pensamiento y el lenguaje de los niños.

Piaget y su equipo consolidaron, con base en un sinnúmero de investigaciones empíricas especializadas, una teoría del desarrollo ontogenético de las estructuras cognitivas, que por su parte inspiró una larga serie de investigaciones comparativas de las sociedades de todos los tiempos y lugares, que confirma las ideas esbozadas por los psicólogos del desarrollo hace setenta años y asumidas por Elias desde la elaboración de sus primeros libros.

La atmósfera intelectual de finales del siglo XIX y de los primeros decenios del XX se hallaba profundamente impregnada del tema de los primitivos. Este se había constituido en un verdadero reto para la comprensión de la condición humana, de la historia, del conocimiento, del comportamiento la conducta, es decir, de todo cuanto concierne a los asuntos humanos. Por ello resulta inadmisible la idea de que justamente Elias se habría mantenido al margen y que sobre un tema tan central se hubiera inventado las cosas (BLOK, 1982; GOODY, 2002, p. $03,04,06)$. Pero resulta insostenible el supuesto de que sobre las sociedades más simples Elias no tenía idea, además por las referencias tempranas a LévyBruhl (WEILER, 2008; 2009). Por otra parte la antropología fue uno de los campos 
de estudio de Elias en Heidelberg. Años después, llegado a su exilio londinense, Elias se ofreció a enseñar antropología a nivel superior. Por lo visto, tenía la idea de tener la competencia adecuada para hacerlo, sabiendo que se encontraba en un país de antropólogos destacados. No parece haber enseñado cursos de antropología finalmente. Pero fue docente en Londres entre 1944 y 1953 de cursos de extensión sobre psicología, psicología social, sociología, relaciones internacionales y economía (DLA, A: Elias, 160). En ellos integraba una bibliografía que muestra que Elias estaba pendiente de los desarrollos en el campo de la antropología. Entre los antropólogos que figuran en los programas de sus cursos, se encuentran autores como Margared Mead, Bronislaw Malinowski, Ruth Benedict, Robert Lowie, G. P. Murdock, H. J. Peake, Richard Thurnwald, Lucien Lévy-Bruhl y muchos otros, todos ellos ciertamente en la más natural compañía de representantes de las diversas corrientes de la psicología y otras disciplinas.

Por el papel que llegaron a jugar para la revolución ontogenética las investigaciones de Jean Piaget y el equipo liderado por él, es de interés registrar que, entre los psicólogos que figuran en las bibliografías de los cursos de extensión de Elias en el periodo mencionado, está presente Piaget. Los libros que de Piaget usaba Elias con regularidad en la docencia son Language and Thought of the Child (1923), The child's conception of the World (1926), The child's conception of Physical causality (1927) y The Moral Judgment of the Child (1930). Menciono los títulos tal como aparecen en los programas de Elias porque en Londres se usaban las traducciones.

Referencias de Elias a Piaget también se encuentran en manuscritos y bocetos. A mediados de los años sesenta, Elias estaba trabajando en una obra que pensaba como de fundamentación de las ciencias sociales. El título provisional era On the development of Human Thought. De los preparativos para esta obra formaban parte diversos avances sobre el pensamiento de Lévy-Bruhl. Es en relación con éste que Elias menciona a Piaget. Elias ve los trabajos de Lucien Lévy-Bruhl sobre la mentalidad primitiva como contribución a una serie de problemas psicológicos.

3. “(...) que havia sido tomada por muitos dos representantes de uma abordagem de desenvolvimento para a psicologia, acima de tudo por Piaget (...)" [tradução dos editores].
(...) which had been taken up by many of the representatives of a developmental approach to psychology above all by Piaget. (Draft to an Introduction to Lévy-Bruhl.DLA, Fondo A: Elias, Lévy-Bruhl etc. 1, L-B.) ${ }^{3}$

Es una breve pero explícita referencia a la psicología evolutiva comentada en páginas anteriores de este ensayo, y de una vez resalta a Piaget. En la siguiente nota, Elias reúne áreas de investigación que vimos integrados también en esta misma psicología. 
In more recent times studies in the thought processes of simpler people have been further advanced both on the basis of studies of children and of the great mass of new evidence about simpler people themselves by men like K. (sic) Werner. (Draft to an Introduction to Lévy-Bruhl. DLA, Fondo A: Elias, Lévy-Bruhl etc. 1, L-B. 1) ${ }^{4}$

No hay duda, aquí Elias se refiere a Heinz Werner, de cuyo libro tenía una edición en inglés en su biblioteca en Inglaterra. Partes de esta biblioteca se han conservado en el mismo archivo en el que reposan los papeles. Por lo demás, hay constancia de que Elias poseía el libro que he comentado ampliamente en las listas que registraron los libros del trasteo de retorno al viejo continente. Agrego otra cita más que encontré entre los borradores que Elias produjo preparando un libro sobre Lévy-Bruhl:

An example that comes to mind, are Piaget's investigations in the forms of thinking of children. He was able to show that the way in which children link experiences to each other is in specific ways different from what are generally regarded as the standard connection between experiences established in those forms of thinking which one traditionally calls rational. What Levy Brühl (sic) tried to do with regard to the patterns of thinking in simpler societies was very similar to what Piaget attempted to do with regard to the patterns of thinking of children.(DLA, A: Elias, Lévy-Bruhl etc. 6/1, L.B. 16) ${ }^{5}$

Lo que pasa es que Elias, siempre que asomaban las críticas contra su teoría, solía responder que la base de la teoría de la civilización psicogenética se encuentra en la psicología infantil (OESTERDIEKHOFF, 2000, p. 16). Así lo hizo también cuando fue acusado de posturas colonialistas y etnocentristas, en 1981 en Amsterdam (WILTERDINK, 1984, p. 298). Quizás el público no entendía a qué Elias se refería o creía que éste estaba improvisando.

Todo lo anteriormente expuesto no significa que Elias haya estado de acuerdo con los psicólogos del desarrollo en todo ni que daba por resuelta la tarea de una nueva epistemología. No es difícil detectar divergencias incluso con Piaget. Pero éstas no pueden entrar en el campo de visión ni convertirse en objeto de reflexión mientras se crea que Elias emprendió sus investigaciones en un mundo apartado del programa de la epistemología genética del gran suizo y mientras perdure la convicción de que se habría enterado de éste solo a posteriori.
4. "Em tempos mais recentes tem avançado os estudos sobre os processos de pensamento de pessoas mais simples. Este avanço tanto se baseia em estudos desenvolvidos com crianças quanto na grande massa de novos indícios referentes às pessoas mais simples, tal como o homem K (sic) Wener (...)" [tradução dos editores].

5. “Um exemplo que vem à mente são as investigações de Piaget sobre as formas de pensamento das crianças. Ele foi capaz de mostrar que a maneira como as crianças vinculam experiências umas com as outras são formas específicas, diferentes do geralmente é considerado como o padrão de conexão entre as experiências, o qual se refere ao que tradicionalmente se denomina formas racionais do pensamento" [tradução dos editores]. 
Quienes dirigen en el presente su atención sobre la historia de la psicología en el siglo XX pueden tomar en consideración lo transcurrido en el tiempo que aquella generación ya no vivió. ¿Qué arroja la distancia acerca del inmenso proceso de cambio en la psicología del cual se sentían optimistas Stern y sus colegas? Unas pocas notas pueden ilustrar la situación. Los psicólogos René van der Veer y Jaan Valsiner, que en la primera mitad de los años noventa prepararon para el público anglosajón un libro con textos del psicólogo ruso Lew Vygotsky (18961935), registran una interesante experiencia que se debe a que las referencias bibliográficas de Vygotsky, para los estándares de hoy, eran muy deficientes. Para corregir esto, los editores del libro asumieron el trabajo, sumamente dispendioso, de buscar en diversos textos referencias indicadas apenas en forma críptica por Vygotsky y el posible origen de sus citas. Estas búsquedas los obligaron a leer un sinnúmero de libros escritos por los antecesores y contemporáneos de Vygotsky. Su experiencia en arqueología del conocimiento la comentan así:

6. "As publicações que fomos forçados a ler, muitas vezes, eram extremamente valiosas nelas mesmas e, muitas vezes, também, nos perguntamos o quanto a psicologia não se desenvolveria se parássemos todas as novas pesquisas e experimentos para refletir e elaborar a respeito dos tesouros encontrados em arquivos da própria psicologia" [tradução dos editores].
(...) the publications we were forced to read were often extremely valuable in their own right and we have often wondered how far psychology would develop if we stopped all new and experimental research for several years and instead reflected upon and elaborated the treasures found in psychological archives (VAN DER VEER \& VALSINER, 1994, p. I). ${ }^{6}$

Del conjunto bibliográfico al que se refieren quienes esto formulan, forman parte los textos que he comentado a modo de ejemplos en los anteriores apartados. Han pasado ya quince años desde la insinuación arriba citada que encierra una idea evidentemente crítica sobre la psicología de finales del siglo XX. Culminado el primer decenio del nuevo milenio se advierten tonos aun más agudos en la comparación de los dos momentos de referencia, los 1930 y el presente, en la evolución de la psicología. La siguiente apreciación es de 2010:

(...) there is increasing evidence that theories and methodology of psychology, especially that of Continental Europe, were far more advanced 60 years ago than what is considered to be our contemporary mainstream psychology.

Before World War II two general schools of thinking in psychology, Northamerican and German-Austrian, could be differentiated. The latter was considerably more sophisticated in its theoretical and phenomenological practices than its North-American offspring. A number of crucial perspectives became lost in psychology when the center of dominance 
in research moved westward over the Atlantic in conjunction with the tragedies of World War II. (VALSINER \& TOOMELA, 2010, p. VIII) ${ }^{7}$

Lo que en concepto de los autores citados se ha perdido en la orientación principal adoptada por la psicología a partir de 1940, coincide con los elementos que Stern resaltaba como signos del gran cambio en la psicología para 1930. Y esta apreciación ha comenzado a tomar forma entre los psicólogos que ven con preocupación el estado actual de su disciplina. Por lo general, éstos son los mismos que propugnan por la recuperación de la psicología en función de la comprensión del desarrollo de la cultura humana a lo largo de la historia.

¿Cuáles son los problemas que se resaltan y qué se sugiere recuperar? Se ha perdido de vista el problema del desarrollo. El signo más claro de ello es la eliminación casi completa de la dimensión temporal del estudio de los fenómenos psíquicos (GILLESPIE, ZIRTOUN, 2010). La perspectiva del desarrollo por lo general se despacha alegando que el desarrollo no es lineal y que la historia es variopinta y rica. Como si la idea fuera original, cuando los primeros en saber que el desarrollo no es lineal son los que se ocupan del desarrollo. "That development is a non-linear process is no big news for developmental scientists" (VALSINER y TOOMELA, 2010, p. VIII). El hecho es que la tendencia dominante en psicología privilegia la evaluación de unos resultados, frente a los procesos dinámicos que han llevado a unos resultados (VALSINER, MOLENAAR, LYRA \& CHAUDARY, 2009). La psicología del gran cambio se atribuía el mérito de haber superado este problema. Se pensaba también haber demostrado que el problema del desarrollo, entendido en términos de cambios en la organización global de las estructuras psíquicas, no es de naturaleza cuantitativa. La psicología predominante desde hace sesenta años, sin embargo, se devolvió a la ilusión de poder consolidar su prestigio científico a través de la cuantificación. Grandes muestras y generalizaciones estadísticas han reemplazado la cuidadosa mirada sobre los fenómenos estudiados en un caso singular (GIGERENZER et al. 1989; LAMIELL, 2003; MOLENAAR, 2004). La hiper-cuantificación se ha acompañado de la fragmentación de las unidades vivas. Un rasgo distintivo de los avances de los años veinte y treinta era justamente lo contrario, la consolidación del estudio de la persona como un todo (KREPPNER, 2010). Finalmente, se registra la reducción del valor de una teoría general a favor de la acumulación de datos, cosa que condujo a una ciencia en gran medida pseudo-empírica, que tiende a emplear grandes esfuerzos y recursos tecnológicos a demostrar lo sabido y comprobar lo evidente (SMEDSLUND, 1995).
7. “Há crescentes evidências de que as teorias e metodologias da psicologia, especialmente da Europa continental, estavam muito mais avançadas, a 60 anos, do que a nossa principal corrente da psicologia contemporânea.

Antes da Segunda Guerra Mundial, duas escolas gerais de pensamento em psicologia, a norteamericana e alemãaustríaca, poderiam ser diferenciadas. A última era consideravelmente mais avançada em suas teorias e práticas fenomenológicas que as suas descendentes norte-americanas. Uma série de perspectivas cruciais se perderam em psicologia quando a hegemonia da pesquisa se moveu para o oeste, cruzando o Atlântico, em conjunto com as tragédias da II Guerra Mundial" [tradução dos editores]. 


\section{Conclusiones}

La psicología del desarrollo, en la forma en que ésta se articuló en los decenios previos a la Segunda Guerra Mundial y que posteriormente fue marginada, ofreció conocimientos que integran los fundamentos sobre los cuales Norbert Elias concibió una ciencia procesual de los seres humanos. Tal ciencia, de acuerdo con Elias, dependería esencialmente de la conceptualización del hombre-sujeto como proceso.

Esto indica la perspectiva en que ha de leerse lo expuesto sobre el papel que para Elias jugó la psicología del desarrollo, y de cantera la etnografía. En adelante, la lectura por lo demás se va a beneficiar del hecho que sus observaciones dispersas sobre las dificultades de concebir al hombre como proceso se han confirmado y precisado. Podemos indicar brevemente la dirección en que esto señala: El bloqueo que, de acuerdo con Elias dificulta el surgimiento de una auténtica ciencia sociológica porque se opone con fuerza a la conceptualización del hombre como proceso, y por tanto, a la interpretación de los datos en una lógica procesual se registra todavía en el nivel que se identifica con el cuarto estadio del modelo del desarrollo cognitivo de Piaget. En el modelo del desarrollo cognitivo piagetiano, este estadio se caracteriza por las operaciones formales que, gracias a la competencia de representación simbólica independizada de la presencia real de los objetos, implicarían la posibilidad de integrar en nuestras representaciones simbólicas una cantidad ilimitada de aspectos (factores). En una palabra, no se ve en el modelo de Piaget razón para el bloqueo que veía Elias y que ciertamente ve y, además, logra explicar Dux. De hecho Elias, cuando se refería a las dificultades de admitir el proceso psíquico (individual como histórico-colectivo) como objeto legítimo de estudio, apuntaba a cierto tipo de causalidad (WEILER, 2008; VAN BENTHEM VAN DEN BERGH, 2012). Sugiere esto que encontró cierto tipo de causalidad ligado con cierta forma también de objeto y que a ambos los veía como característica del punto en el desarrollo del pensamiento en que diagnosticaba un bloqueo.

Si se consideran las reflexiones de Elias sobre este punto a la luz de la teoría histórico-genética, que incluye la reconstrucción de las más tempranas estructuras cognitivas, a partir de las condiciones evolutivas de nuestra especie - asunto enfatizado por Elias en la Teoría del Símbolo - se nota que se refería a unas estructuras categoriales que todos los seres humanos forman en su más temprana ontogénesis y que aun en las sociedades industriales que han desarrollado las operaciones formales no han sido superadas del todo. Una sociología procesual en esta perspectiva, se entiende condicionada por el desarrollo de una lógica procesual, que implica la superación de los restos de la lógica subjetivista, y ésta en sus últimos reductos todavía comparte con el mundo antiguamente conformado por causantes animados, la estructura de una relación bipolar en la 
cual todas las formas fenoménicas dadas remiten a algo que de alguna manera ya lo contiene. Tal estructura es la misma en que queda atrapado todo intento de derivar al sujeto de un sujeto, al conocimiento de conocimiento

\begin{abstract}
The main interest of this paper resides in the question about how Norbert Elias faced the doubt of the knowledge that started with the idea that the knowledge do not depends on the subjectivity that builds it up. A totally new theory, as Elias says, demanded the rise of "the subject" producer of the knowledge and all the historical forms of human life, a veil of the inscrutable mystery that was preserved on the philosophical tradition. There was necessary to investigate how and under what conditions empirically questionable, the human beings really acquire the knowledge of the world that serves for their living. This task was assumed, in large-scale, by the psychology of the development formed at the early XX century. So on, the question raised at first in this paper is translated in the question about the relation between cognitive purposes and the strategy of investigation that Elias asks about, with the ontogenetic revolution produced by de psychology of development. The exposition focuses on the period of formation of the idea of development psycho-genetic, axis of the Elias's sociology, that is in history of psychology a period of important advances throughout the problem of human development.

Keywords: Norbert Elias, Subject, Theory of Knowledge, Psychology of development, Onto-genesis, Human Development
\end{abstract}

\title{
Referencias
}

BLOK, A. "Primitief en geciviliseerd", en Sociologische Gids, themanummer. mei/ juni/ juli/ augustus (jg. XXIX), 1982, p. 197-209.

BÜHLER, K. Die geistige Entwicklung des Kindes. Jena. Gustav Fischer Verlag, (1918/ 1922).

DEUTSCH, W. "Die Entdeckung der frühen Jahre: Vergangenheit erinnern und frühe Jahre erforschen", Journal für Psychologie. 12, 1, 2004, p. 11-24.

(DLA) A: Elias, hace referencia al Fondo del archivo Deutsches Literaturarchiv Marbach, que reúne los papeles legados por Elias.

DUX, G. Die Logik der Weltbilder: Sinnstrukturen im Wandel der Geschichte. Frankfurt: Suhrkamp, 1982.

. Historisch-genetische Theorie der Kultur. Weilerswist: Velbrück,

2000. 
- Teoría histórico-genética de la cultura. La lógica procesual en el cambio cultural. Bogotá: Ediciones Aurora, 2012.

ELIAS, N. El proceso de la civilización. México: Fondo de Cultura Económica, 1987.

. Mi Trayectoria Intelectual. Barcelona: Península, 1995a.

. Sociología fundamental, Barcelona: Gedisa, 1995b.

GABRIEL, N. "Norbert Elias and developmental psychology", The Sociological Review, special Issue on Elias, 2011, p. 202-219.

GIGERENZER, G. (et al.) The empire of change. Cambridge. Cambridge University Press, 1989.

GILLESPIE, A. \& ZITTOUN, T. "Styling the Movement of Thought", In: J. Valsiner \& R. Van der Veer, Methodological thinking in psychology: 60 years gone astray? Charlotte: Information Age Publishing, 2010, p. 69-88.

GLEICHMANN, P. R.“Norbert Elias aus Anlass seines 90. Geburtstages”, Kölner Zeitschrift für Soziologie und Sozialpsychologie. H. 2/ 1987, p. 406-417.

GOODY, J. "Elias and the anthropological tradition", Anthropological Theory. Vol. 2(4), 2002, p. 401-212.

."The 'Civilizing Process' in Ghana", Archives européennes de sociologie. Vol. 44 (1), 2003, p. 61-73.

. "Elias et la tradition anthropologique", In: S. Chevalier \& J.-M. Privat (eds.). Norbert Elias et l'anthropologie: 'Nous sommes tous si étranges...'. Paris: CNRS Editions, 2004.

. The Theft of History. Cambridge: University Press, 2006.

JAHODA, G. Images of Savages: Ancient Roots of Modern prejudice in western culture. London: Routledge, 1999.

JOLY, M. Norbert Elias au-delà de Freud. Sociologie, psychologie, psychoanalyse. Paris: La Découverte, 2010.

KILMINSTER, R. Norbert Elias: Post-philosophical sociology. London: Routledge, 2007.

. "Norbert Elias's post-philosophical sociology: from 'critique' to relative detachment", The Sociological Review, special Issue, 2011, p. 91-116. 
KOFFKA, K. Die Grundlagen der psychischen Entwicklung. Verlag A. W. Zickfeld, Osterwieck a.H, 1921.

KÖHLER, W. Intelligenzprüfungen an Anthropoiden. Berlin: Abhandlungen der preussischen Akademie der Wissenschaften, physikalisch-mathematische Klasse, 1917.

KREPPNER, K. "William L. Stern, 1871-1938: A neglected founder of developmental psychology", Developmental Psychology. 28 (4), 1992, p. 539-47.

."Heinz Werner and the Psychological Institute in Hamburg", en Valsiner, Jaan (ed.). Heinz Werner and developmental science. New York, Dordrecht, London, Moscow: Kluwer Academic / Plenum Publisher, 2005, p. 55-74.x

. "Observational Methodology and Film in Developmental Psychology", In: J. Valsiner \& A. Toomela (eds.). Methodological thinking in psychology: 60 years gone astray? Charlotte. Information Age Publishing, 2010, p. 231-259.

KRUEGER, F. Über Entwicklungspsychologie (Arbeiten zur Entwicklungspsychologie 1). Leipzig: W. Engelmann, 1915.

LAMIEL, J. T. Beyond individual and group differences. Thousand Oaks: Sage, 2003.

LANGER, J. "Werner's Comparative Organismic Theory", In: Mussen, Paul H. (ed.).Carmichael's Manual of Child Psychology. Third Edition, Vol. I. New York, London, Sydney, Toronto: John Wiley \& Sons, 1970, p. 733-771

LISTON, K. \& MENNELL, S. "III Met in Ghana: Jack Goody and Norbert Elias on Process and Progress in Africa", Theory, Culture and Society 26 (7-8), 2009, p. 52-70.

MENNELL, S. Norbert Elias - an introduction. Dublin: University College Dublin Press, 1992.

MOLENAAR, P. C. M. "A manifesto on psychology as idiographic science: Bringing the person back into scientific psychology, this time, forever", Measurement: Interdisciplinary research and perspectives 2. 2004, p. 201-218.

MÜLLER, U. "The context of the formation of Heinz Werner's ideas", In: Valsiner, Jaan (ed.). Heinz Werner and Developmental Science. New York, Dordrecht, London, Moscow. Kluwer Academic / Plenum Publisher, 2005.

NIESTROJ, B. H. E. "Norbert Elias: A milestone in historical psychosociology.The making of the social person". Journal of Historical Sociology, vol. 2 (2), 1989, p. 136-160. 
OESTERDIEKHOFF, G. W. Zivilisation und Strukturgenese. Norbert Elias und Jean Piaget im Vergleich. Frankfurt: Suhrkamp, 2000.

- "La sociología genético-estructural como heredera de la sociología clásica y de la teoría de la civilización", In: Vera Weiler (ed.), Norbert Elias y el problema del desarrollo humano. Bogotá: Ediciones Autora, 2011, p. 71-95.

SMEDSLUND, J. "Psychology: Common sense and the pseudoempirical", In: J. Smith; R. Harré \& . L. Langehove (eds.). Rethinking psychology. London: Sage, 1995, p. 196-206.

STERN, C. \& STERN, W. Die Kindersprache. Eine psychologische und sprachtheoretische Untersuchung. Leipzig: Verlag Johann Ambrosius Barth, 1907.

STERN, W. Person und Sache. System der philosophischen Weltanschauung. (Vol. I, Ableitung und Grundlehre). Leipzig: J.A. Barth, 1906.

STERN, W. Psychologie der frühen Kindheit bis zum sechsten Lebensjahre. Leipzig: Quelle \& Meyer, 1930.

VALSINER, J. Developmental Psychology in the Soviet Union. Bloomington, Indianapolis. Indiana University Press, 1988.

(ed.) Heinz Werner and Developmental Science. New York, Dordrecht, London, Moscow. Kluwer Academic / Plenum Publisher, 2005.

VALSINER, J. \& TOOMELA, A. (eds.) Methodological thinking in psychology: 60 years gone astray? Charlotte: Information Age Publishing, 2010.

VALSINER, J.; MOLENAAR, P.; LYRA, M. C.F.P. \& CHAUDARY, N. (eds.) Dynamic process methodology in the social and developmental sciences. New York: Springer, 2009.

VAN BENTHEM VAN DEN BERGH, G. Attribution of blame as the past and present mode of orientation: the social sciences as a potential improvement, http:// www.norberteliasfoundation.nl/docs/pdf/BlameAttribution.pdf, 2012.

VAN DER VEER, R. "The making of a developmental psychologist", en Valsiner, Jaan (ed.), Heinz Werner and developmental science. New York, Dordrecht, London, Moscow: Kluwer Academic / Plenum Publisher, 2005, p. 75-105.

VAN DER VEER, R. \& VALSINER, J. Understanding Vygotsky. A quest for synthesis. Oxford \& Cambridge: Blackwell, 1993. 
. The Vygotsky Reader. Oxford \& Cambridge. Blackwell, 1994.

VOLKELT, H. Über die Vorstellungen der Tiere. Leipzig, Berlin: Verlag Wilhelm Engelmann, 1914.

WEILER, V. "Lucien Lévy-Bruhl visto por Norbert Elias", Revista Mexicana de Sociología. 70. n. 4 (octubre-diciembre), 2008, p. 791-822.

- "Algunos antecedentes de la estrategia de investigación de Norbert Elias", In: Carina V. Kaplan y Victoria Orce (coords.). Poder, Prácticas Sociales y Proceso Civilizador. Buenos Aires: NOVEDUC, 2009, p. 33-42.

. "La versión psicogenética de la Historia Cultural. A propósito de los cien años del Instituto de Historia Cultural y Universal en Leipzig", en Anuario Colombiano de Historia Social y de la Cultura. Vol. 37. N. 1, 2010, p. 227-267.

. "El problema del desarrollo en la psicología hasta 1940 en relación con el pensamiento de Norbert Elias", en Vera Weiler (ed.), Norbert Elias y el problema del desarrollo humano. Bogotá: Ediciones Aurora, 2011, p. 97-134.

WERNER, H. Einführung in die genetische Entwicklungspsychologie. 3a ed., 1953. Muniche: J.A. Barth, 1926. [Trad., Compendio de Psicología Evolutiva. Barcelona. Salvat., 1936]; [Trad., Psicología comparada del desarrollo mental. Buenos Aires: Paidós, 1965]

. Comparative Psychology of Mental Development. New York, London: Harper \& Brothers, 1940.

WILTERDINK, N. "Die Zivilisationstheorie im Kreuzfeuer der Diskussion. Ein Bericht vom Kongress über Zivilisationsprozesse in Amsterdam", In: Gleichmann, P., Goudsblom, J. \& Korte, H. (eds.). Macht und Zivilisation. Materialien zu Norbert Elias Zivilisationstheorie 2. Frankfurt: Suhrkamp, 1984, p. 280-404.

WUNDT, W. Elemente der Völkerpsychologie. Grundlinien einer psychologischen Entwicklungsgeschichte der Menschheit. Leipzig: Alfred Kröner, 1912. 